\title{
Analysis of H-plane Circular Bend of Rectangular Waveguide based on Equivalent Multi-transmission Line Network Representation
}

\author{
Hsu,Jui-Pang, Tetsuo Anada and Takaharu Hiraoka
}

Faculty of Engineering,Kanagawa University Yokohama, Japan 221

\begin{abstract}
In order to calculate the wide-band frequency characteristics of the H-plane circular bend of rectangular waveguide with an arbitrary bend curvature including sharp bend and any bend angle, a systematic method based on planar circuit theory is proposed; equivalent multi-transmission line model are formulated and successfully applied to the practical structure, as well as E-plane circular bend case. [7] .
\end{abstract}

\section{Introduction}

H-plane circular bend of the rectangular waveguide shown in Fig. 1 , is an important waveguide component. However, so far obtained analytical method and its numerical results [1-5] are limited to the gradual bend case only. Therefore, analytical method and numerical data which can cover wide bend parameters (=bend curvature $\mathrm{C}$ and bend angle $\alpha$ which are explained in Fig. 1) including sharp bend are strongly needed not only for academic interest but also for the integration of microwave circuit.

This paper treats the above problem based on the equivalent multi-transmission line network representation [6], which is derived from H-plane planar circuit equations [8]. Practically, how to calculate the network parameters is explained in general, based on planar circuit theory and then actual network parameters are calculated as a function of normalized frequency with bend curvature parameter C. Finally, based on these parameters, the frequency characteristics for right angle H-plane circular bend case is calculated for wide range of bend curvature as an example.

\section{Equivalent multi-transmission line network representation}

$\mathrm{H}$ - plane curved rectangular waveguide $(\mathrm{d} \times \mathrm{W})$ can be considered to be a H-plane planar circuit as a whole, where field components and planar voltage/current are related by $(B),(C),(D)$ in Table 1 [8].

Hence, the frequency characteristics of the curved rectangular waveguide shown in Fig. 1 can be given by solving planar circuit equations $(\mathbf{E}, \mathbf{G})$ in Table 1 directly undex the short circuited boundary condition (F) and TE10 dominant mode excitation condition.

Here, instead, the frequency characteristics are calculated based on the equivalent multi-transmission line network representation given in Fig. 2. This equivalent network representation is derived by the modal analysis of the planar circuit, appling the separation

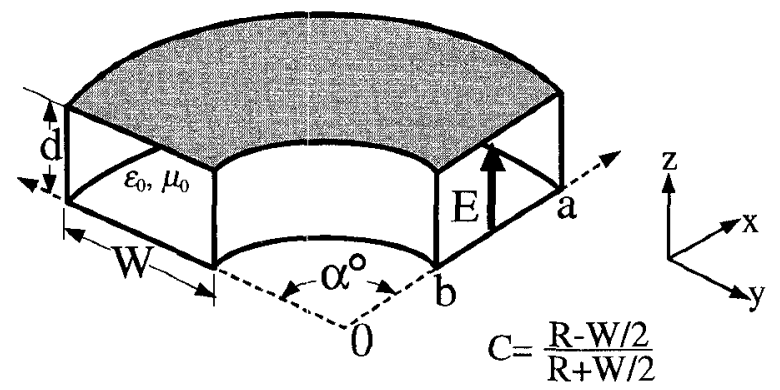

$\mathbf{C}=\frac{\mathbf{b}}{\mathbf{a}}$ :bend curvature parameter

( $R$ : average radius of the curved waveguide)

Fig.1 H-plane $\alpha^{\circ}$ circular bend of rectangular wave-guide and coordinate system

\begin{tabular}{|l|c|c|}
\hline Field & $\mathbf{E}\left(0, E_{z}\right), \mathbf{H}\left(\mathbf{H}_{i}, 0\right)$ & $\mathbf{B}$ \\
\hline $\begin{array}{l}\text { Field } \\
\text { component }\end{array}$ & $\begin{array}{l}E_{z}(x, y, z)=E_{z}(x, y) \\
\mathbf{H}_{\mathrm{t}}(x, y, z)=\mathbf{H}_{\mathrm{t}}(x, y)\end{array}$ & $\mathbf{C}$ \\
\hline $\begin{array}{l}\text { Electric } \\
\text { Voltage } \\
\text { Electric } \\
\text { Current }\end{array}$ & $V^{\mathrm{E}}=-E_{\mathrm{z}}(x, y) \cdot d \quad[\mathrm{~V}]$ & $\mathbf{D}$ \\
\hline $\begin{array}{l}\text { Planar } \\
\text { Circuit } \\
\begin{array}{l}\text { Equations } \\
\text { with B. C. }\end{array}\end{array}$ & $\left\{\begin{array}{c}\operatorname{grad} V^{\mathrm{E}}=-j X^{\mathrm{E}} \mathbf{J}^{\mathrm{E}} \\
\operatorname{div} \mathbf{J}^{\mathrm{E}}=-j B^{\mathrm{E}} \mathrm{V}^{\mathrm{E}}\end{array}\right.$ & $\mathbf{E}$ \\
\hline $\begin{array}{l}V^{\mathrm{E}}=0\left(\mathrm{or} \mathbf{J}^{\mathrm{E}} \times \mathbf{n}=0\right) \\
\text { Immittancc }\end{array}$ & $X^{\mathrm{E}}=\omega \mu_{0} d, B^{\mathrm{E}}=\frac{\omega \varepsilon_{0}}{d}$ & $\mathbf{F}$ \\
\hline $\begin{array}{l}\text { propagation } \\
\text { constant }\end{array}$ & $\beta_{t}^{\mathrm{E}}=k_{0}=\omega \sqrt{\varepsilon_{0} \mu_{0}}$ & $\mathbf{H}$ \\
\hline
\end{tabular}

Table1 H-plane planar circuit equations and the relation between field components and planar voltage/current with boundary condition. 
of variable technique to the planar circuit structure (Table 2 [6]).

In the straight rectangular waveguide section ( $A_{1}$ in Table 2 ), where $1-s-z$ coordinate system is defined, $p$-th mode voltage and current are given by $\left(\mathrm{B}_{1}\right)$, where $\mathrm{p}$-th width mode $f_{p}^{E}(s)$ is also defind; modal voltage and modal current along $\ell, V_{\mathrm{p}}(\ell)$ and $I_{\mathrm{p}}(\ell)$, are retated by the transmission line equations (C1) with p-th mode characteristic impedance $Z_{c p}^{\prime E}$ and propagation constant $\beta_{p}^{E}$.

In the curved rectangular waveguide section ( $\mathrm{A}_{2}$ in Table 2), where $r-\theta-z$ coordinate system is defined, $\mathrm{p}$-th mode voltage and current are given by $(\mathrm{B} 2)$, where $n$-th radial mode function $R_{n}^{E}(r)$ is defined by $(A)$ in Table 3 ; the modal voltage and modal current along $\theta, V_{n}^{E}(\theta)$ and $I_{n}^{E}(\theta)$, are related by the transmission-line-like equations $\left(\mathrm{C}_{2}\right)$ with $\mathrm{n}$-th mode characteristic impedance $Z_{c n}^{E}$ and circular (azimuthal) propagation constant $v_{n}^{E}$. The coupling between $\mathrm{p}$-th mode in straight waveguide and $\mathrm{n}$-th mode in the circular bend is defined by ideal transformer ratio and given by eq.(1)

$$
\mathrm{n}_{n, p}^{(i)}=\frac{1}{W} \int_{0}^{W} f_{p}^{E}\left(s^{(i)}\right) R_{n}^{E}(r) d s^{(i)} \quad(i=\# 1, \# 2)
$$

Hence, the whole equivalent network becomes that in Fig.2. Also 2-port (input/output) mode impedance matrix is given by eq.(2)

$\mathbf{Z}_{p, q}^{E}=\sum_{n=1}^{\infty}\left(\begin{array}{cc}n_{n p}^{(1)} & 0 \\ 0 & n_{n p}^{(2)}\end{array}\right) \cdot\left(\begin{array}{cc}Z_{c n}^{E} \operatorname{coth} j v_{n} \alpha & Z_{c n}^{E} \operatorname{csch} j v_{n} \alpha \\ Z_{c n}^{E} \operatorname{csch} j v_{n} \alpha & Z_{c n}^{E} \operatorname{coth} j v_{n} \alpha\end{array}\right) \cdot\left(\begin{array}{cc}n_{n q}^{(1)} & 0 \\ 0 & n_{n q}^{(2)}\end{array}\right)$

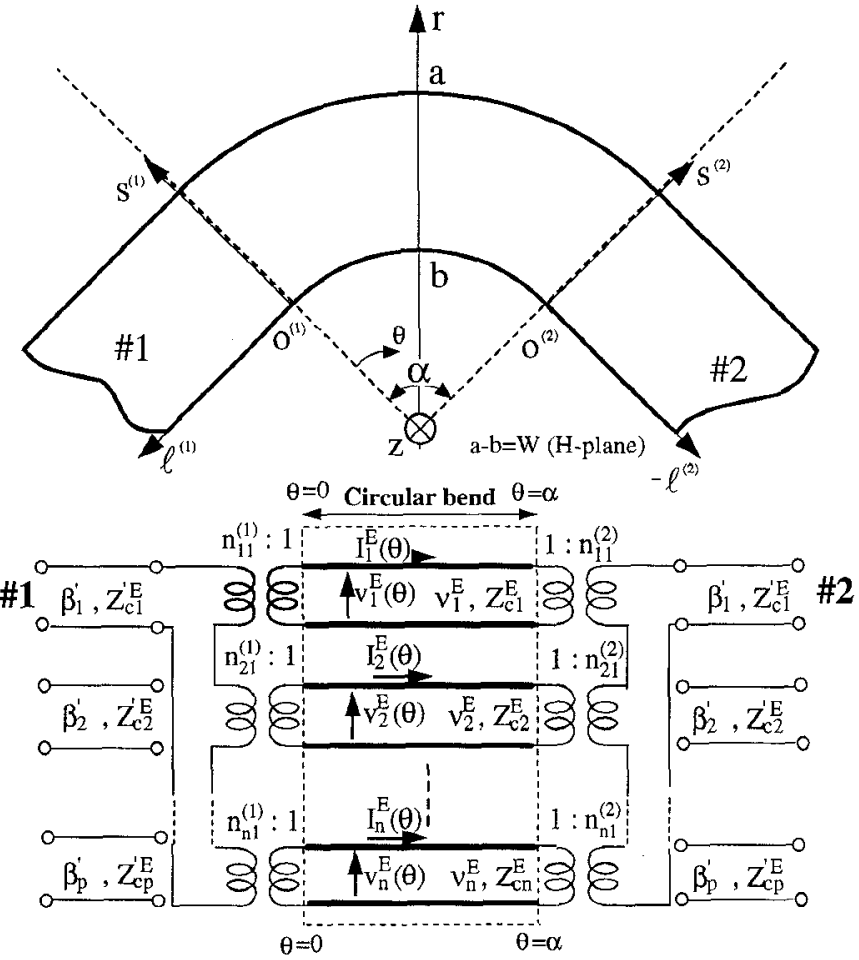

Fig.2 Coordinate system and equivalent multi-transmission line network

Table 3 Radial normal mode function for H-plane circular bend section.

\begin{tabular}{|c|c|c|c|}
\hline Straight waveguide $(\ell, s, z)$ & & Circular bend $(r, \theta, z)$ & \\
\hline waveguide straight section & $\mathbf{A}_{1}$ & waveguide circular bend section & 12 \\
\hline $\begin{array}{l}\text { Separation of variable with } \\
p \text {-th mode width function } f_{p}^{E}(s)\end{array}$ & & $\begin{array}{l}\text { Separation of variable with } R_{n}^{E}(r) \\
n \text {-th radial mode function }\end{array}$ & \\
\hline $\begin{array}{l}V_{p}^{\mathrm{E}}(\ell, s)=V_{p}^{\mathrm{E}}(\ell) f_{p}^{\mathrm{E}}(s) \\
J_{\ell p}^{\mathrm{E}}(\ell, s)=\left[I_{p}^{\mathrm{E}}(\ell) / W\right] f_{p}^{\mathrm{E}}(s) \\
J_{s p}^{\mathrm{E}}(\ell, s)=j \frac{1}{X^{\mathrm{E}}} V_{p}^{\mathrm{E}}(\ell) f_{p}^{\mathrm{E}}(s) \\
f_{p}^{\mathrm{E}}(s)=\sqrt{2} \sin (p \pi s / W)(p=1,2, \cdots)\end{array}$ & $\mathbf{B}_{1}$ & $\begin{array}{l}V_{n}^{\mathrm{E}}(r, \theta)=V_{n}^{\mathrm{E}}(\theta) \cdot R_{n}^{\mathrm{E}}(r) \\
J_{\theta n}^{\mathrm{E}}(r, \theta)=I_{n}^{\mathrm{E}}(\theta) \cdot R_{n}^{\mathrm{E}}(r) / r \\
J_{m}^{\mathrm{E}}(r, \theta)=j \frac{1}{X^{\mathrm{E}}} V_{n}^{\mathrm{E}}(\theta) \cdot R_{n}^{\mathrm{E}}(r) \\
R_{n}^{\mathrm{E}}(r): \text { see Table } 3(n=1,2, \cdots)\end{array}$ & $\mathbf{B}_{2}$ \\
\hline Transmission line equations along $\ell$ & & Transmission line equations along $\theta$ & \\
\hline $\begin{array}{l}\frac{d V_{p}^{\mathrm{E}}}{d \ell}=-j X_{p}^{\mathrm{E}} I_{p}^{\mathrm{E}}, X_{p}^{\mathrm{E}}=\frac{X^{\mathrm{E}}}{W} \\
\frac{d I_{p}^{\mathrm{E}}}{d \ell}=-j B_{p}^{\mathrm{E}} V_{p}^{\mathrm{E}}, \quad B_{p}^{\mathrm{E}}=\frac{\left(\beta_{p}^{\mathrm{E}}\right)^{2}}{X^{\mathrm{E}}} W \\
Z_{c p}^{\mathrm{E}}=\frac{X^{\mathrm{E}}}{\beta_{p}^{\mathrm{E}} W}=\frac{\omega \mu_{0} d}{\beta_{p}^{\mathrm{E}} W} \\
\beta_{p}^{\mathrm{E}}=\sqrt{k_{0}^{2}-(p \pi / W)^{2}}\end{array}$ & $\mathrm{C}_{1}$ & $\begin{array}{l}\frac{d V_{n}^{\mathrm{E}}}{d \theta}=-j X^{\mathrm{E}} I_{n}^{\mathrm{E}} \\
\frac{d I_{n}^{\mathrm{E}}}{d \theta}=-j \frac{\left(v_{n}^{\mathrm{E}}\right)^{2}}{X^{\mathrm{E}}} V_{n}^{\mathrm{E}} \\
v_{n}^{\mathrm{E}}: \text { circular propagation constant } \\
Z_{c n}^{\mathrm{E}}=\frac{X^{\mathrm{E}}}{v_{n}^{\mathrm{E}}}\end{array}$ & $\mathrm{C}_{2}$ \\
\hline
\end{tabular}

\begin{tabular}{|c|c|}
\hline$R_{n}^{E}: n$-th radial normal mode & \\
\hline $\begin{array}{l}\frac{1}{r} \frac{d}{d r}\left(r \frac{d R_{n}^{E}}{d r}\right)+\left[\left(\beta_{t}^{E}\right)^{2}-\frac{\left(v_{t}^{E}\right)^{2}}{r^{2}}\right] R_{n}^{E}=0 \\
\beta_{t}^{E}=k_{0} \\
\text { B.C. } \quad R_{n}^{E}=0 \quad(r=\mathrm{a}, \mathrm{b}) \\
\int_{\mathrm{b}}^{\mathrm{a}} \frac{R_{m}^{E}(r) R_{n}^{E}(r)}{r} d r=\delta_{m n}\end{array}$ & $\mathbf{A}$ \\
\hline Analytical solution for $\beta_{i}^{\mathrm{B}}\left(=k_{0}\right)=0$ & \\
\hline $\begin{array}{l}R_{n}^{E}(r)=\sqrt{-\frac{2}{\ln C}} \sin \left[\frac{n \pi}{\ln C} \ln \frac{r}{a}\right] \\
v_{n}^{E}=-j \frac{\pi}{\ln C} n \quad(n=1,2, \cdots) \\
C=b / a\end{array}$ & $\mathbf{B}$ \\
\hline $\begin{array}{c}\text { Numerical solution for } \beta_{t}^{\mathrm{E}} \neq 0 \text { by } \\
\text { non-uniform transmission line }\end{array}$ & \\
\hline $\begin{array}{l}v(r)=R_{n}^{E}(r), i(r)=j r \cdot \frac{d R_{n}^{\mathrm{E}}}{d r} \\
\left\{\begin{array}{l}\frac{d v}{d r}=-j x(r) i(r) \\
\frac{d i}{d r}=-j b(r) v(r)\end{array}\right. \\
x=1 / r, b=k_{0}^{2} r-v^{2} / r \\
\text { B.C. } v(r)=0(r=\mathrm{a}, \mathrm{b})\end{array}$ & C \\
\hline
\end{tabular}




\section{Calculation of radial normal mode function}

The key step in this analysis is the calculation of radial normal mode function $R_{n}^{E}(r)$ and circular propagation constant $v_{n}^{E}$ defined by (A) in Table 3 as function of operating frequency (or $\beta_{t}=k_{0}$ ) and bend curvature parameter $C$. How to calculate them is explained in the following. When mode voltage and mode current along $r$ are defined in such away as (C) in Table 3 , the second order differential equation (A) in Table 3 can be transformed to a nonuniform transmission line equations given by $(C)$ in Table 3 .

In order to calculate $R_{n}^{E}$ and $v_{n}^{E}$ numerically for a given $\beta_{t}=k_{0}$ or frequency, this non-uniform transmission line between $b$ and $a$ is approximated by cascade connection of infinitesimally small uniform transmission line section as shown in Fig. 3. In our present case, waveguide width $W(=a-b)$ is equally divided into $\mathrm{N}$ sections ; position dependent original reactance $x$ and susceptance $b$ given by (C) in Table 3 are approximated to be the corresponding constant value at the center position of each section, respectively. Then, the corresponding eigenvalue problem is solved by the following way. Assuming $v$, F-matrix of each uniform section $\mathbf{F}_{i}$ can be easily calculated. Therefore, Total F-matrix between port $b$ and port $a$ is given by their cascade product and can be expressed by eq. (3)

$$
\left(\begin{array}{c}
v_{b} \\
i_{b}
\end{array}\right)=\mathbf{F}_{1} \cdot \mathbf{F}_{2} \cdots \cdot \mathbf{F}_{N}\left(\begin{array}{c}
v_{a} \\
i_{a}
\end{array}\right)=\left(\begin{array}{cc}
A_{t} & B_{t} \\
C_{t} & D_{t}
\end{array}\right)\left(\begin{array}{c}
v_{a} \\
i_{a}
\end{array}\right)
$$

Numerical search of $v_{n}^{E}$, which makes $B_{t}=0$, gives $v_{n}^{E}$ and then $R_{n}^{E}(r)$. Thus calculated circular propagation constants $v_{n}^{E}$ vs normalized frequency for wide range of curvature parameter $C$ are shown in Figs. 4 and 5, where the normalized frequency is defined as $F=2 W / \lambda_{0}$ ( $\lambda_{0}=$ free space wavelength). Also radial normal mode functions of lower order are shown in Figs. 4 and 5. Through these calculation $\mathrm{N}$ is taken as 200 or above this value. Analytical solution for $F=0.0\left(\beta_{t}=0\right)$ is exactly given by (B) in Table 3 , which is practically outside desired frequency band but is used for demonstrating the validity of the present numerical analysis.

\section{Frequency characteristics of right angle circular bend}

Once the $R_{n}^{E}(r, C, F)$ are obtained, the ideal transformer ratio $n_{n p}$ defined by eq.(1) are easily calculated; some calculated results are shown in Figs. 4 and 5 for $p=1$. By these calculation, the required network parameters of the equivalent multi-transmission line in Fig. 2 are prepared. Therefore, using these network parameters, frequency characteristics of circular bend can be calculated for any bend angle and bend curvature. As an example, frequency characteristic of the VSWR for the right-angle circular bend case are calculated and shown in Fig. 6 with curvature $C$ as a parameter.

\section{Conclusion}

Analytical method which can calculates input/output performance of H-plane circular bend structure is explained based on the equivalent multi-transmission line network representation and applied to the practical structure. Wide band frequency characteristics for right angle circular bend case are calculated as an example for wide range of curvature parameter.

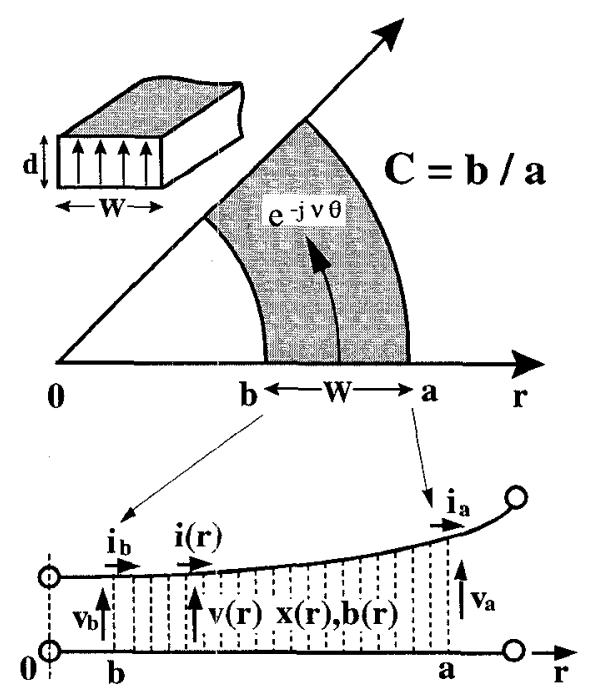

Fig.3 Non-uniform transmission line model and its step-like approximation
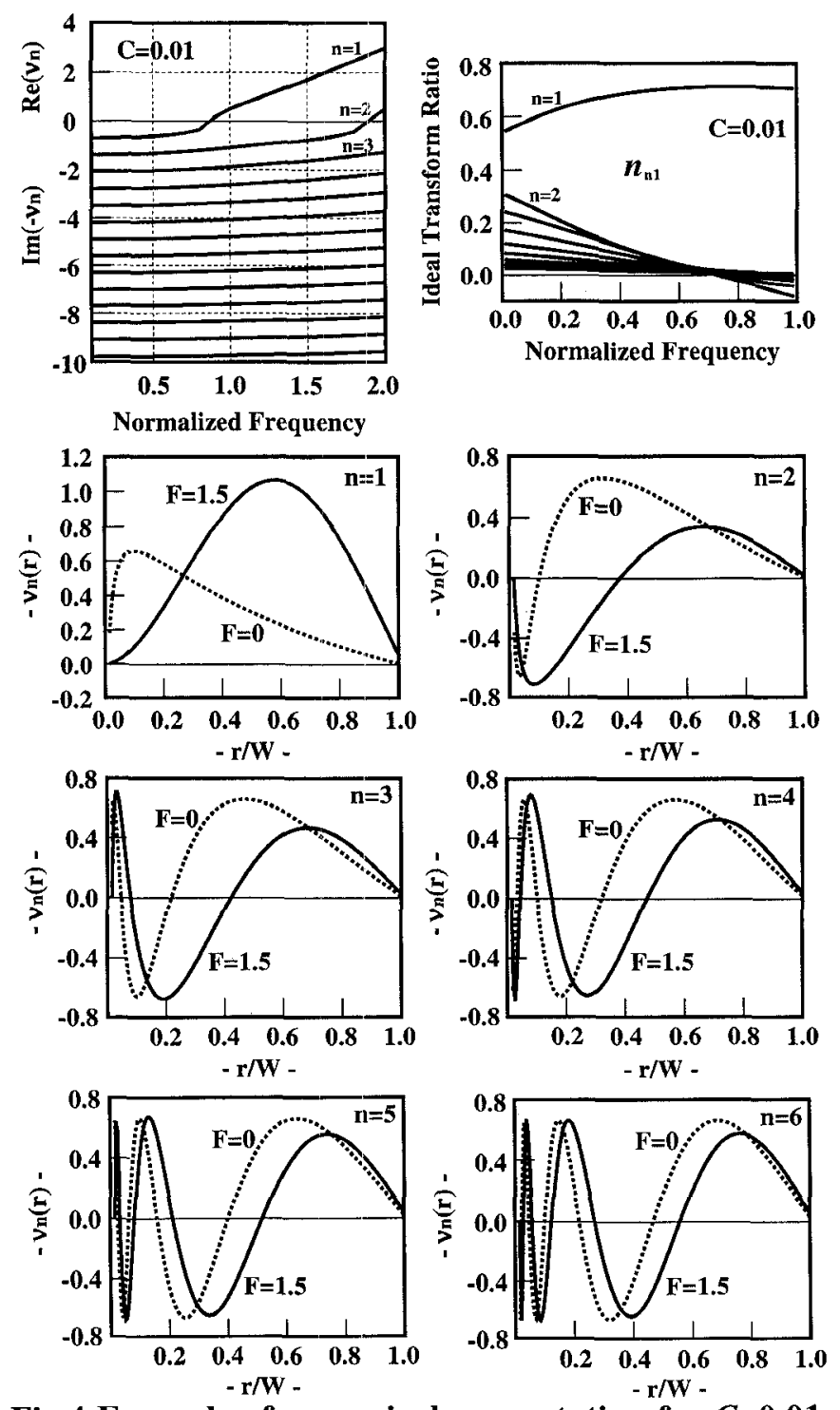

Fig.4 Example of numerical computation for $C=0.01$ based on F-matrix; circular propagation constant, the corresponding radial mode function at $F=0.0$ and $F=1.5$ and the ideal transform ratio $n_{n 1}$ 

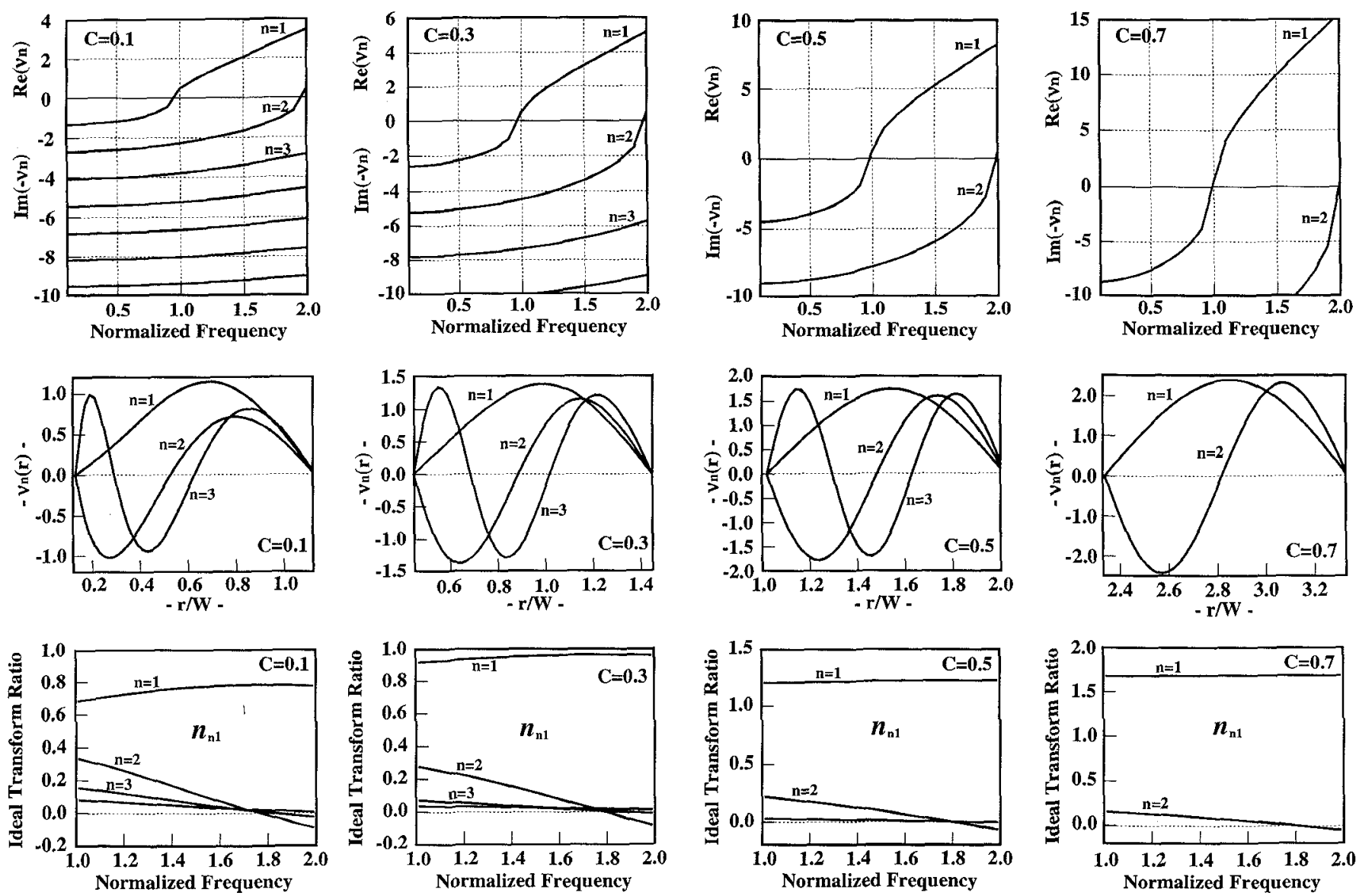

Fig.5 Circular propagation constant $V_{\mathrm{n}}(F, C)$, radial mode function of lower mode $V_{n}=R_{n}(r, C, F=1.5)$ and ideal transformer ratio between $n$-th radial mode and $\mathbf{T E}_{10}$ mode $\left(n=n_{\mathrm{n} 1}\right)$, where curvature parameter $C$ are taken as $0.1,0.3,0.5$ and 0.7 and normalized frequency $F$ is defined by $2 W / \lambda_{0}$. ( $\lambda_{0}=$ free space wavelength)

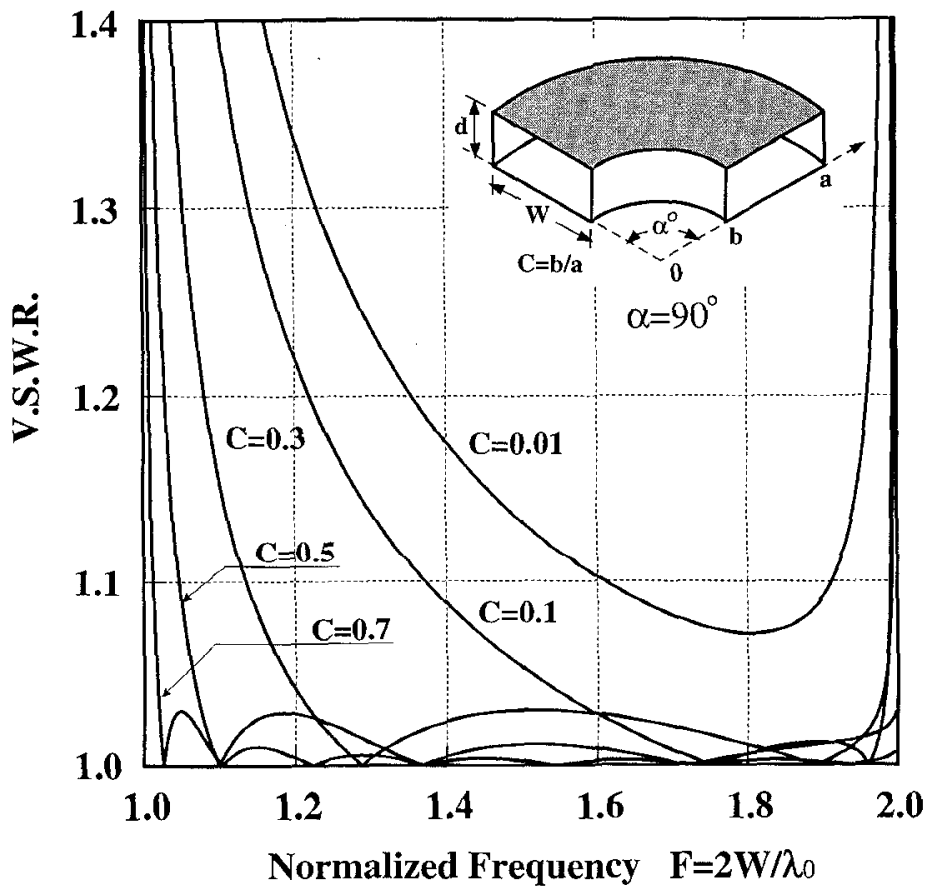

Fig.6 Frequency characteristics of VSWR for right angle H-plane circular bend with bend curvature parameter $\mathbf{C}$.

\section{References}

1. S.O.Rice, "Reflection from circular bends in rectangular waveguidesmatrix theory,", Bell Syst. Tech. J., vol.27, no.2, pp305-349, 1948

2. J.A. Cochran and R.G. Pecina, "Mode propagation in continuously curved waveguides,", Radio Science, vol.1, no.6, pp679-696, June

3. L. Lewin, "Theory of Waveguides,", New York Wiley, 1975 P.L. Carle, "New accurate and simple equivalent circuit for circular Eplane bends in rectangular waveguide", Electronics Letters, vol.23, no.10, pp. 531-532, May 1987.

4. Weisshaar, S.M.Goodnick, V.K.Tripathi,, "A rigorous and efficient method of moments solution for curved waveguide bends,", IEEE MTT vol.40, no.12, pp.2200-2206 Dec. 1992.

5. B.Gimeno, N.Guglielmi, "Multimode network representation for $\mathrm{H}$ and E-plane uniform bends in rectangular waveguide" IEEE MTT-S Digest, TU4B-5 vol.1 (pp.241-244)

6. Hsu,Jui-Pang, T. Anada, "Systematic Analysis Method of E-and Hplane circular bend of Rectangular Waveguide based on the Planar Circuit Equations and Equivalent Network Representation", IEEE MTT-S Digest, WE3F-E1 vol.2 (pp749-752)

7. Hsu,Jui-Pang, T. Anada, "Analysis of E-plane Circular Bend of Rectangular Waveguide based on Equivalent Multi-transmission Line Network Representation", APMC'95 S21-5 pp466-469

8. Hsu,Jui-Pang, "Analysis Method for Electromagnetic wave Problems vol.2 ed. by.E.Yamashita Chap.6", pp226-251, Artech House 